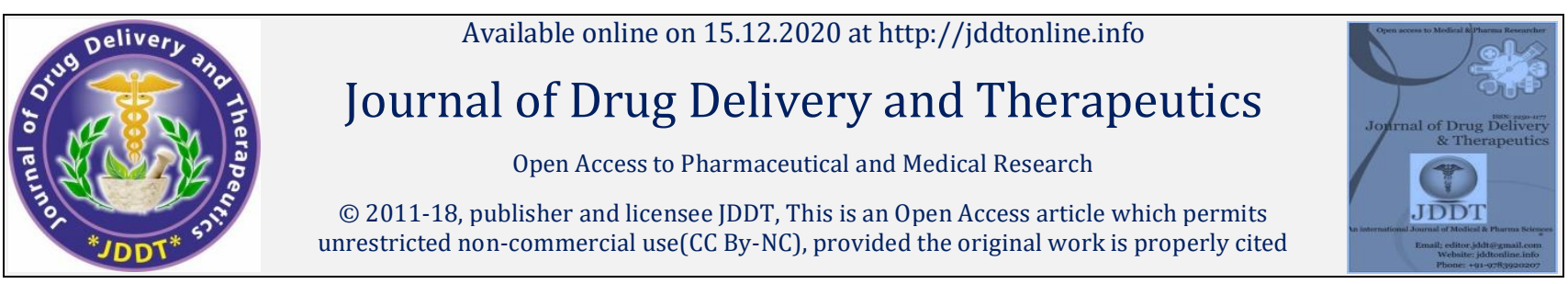

Open $\odot$ Access

Review Article

\title{
An Overview: Preparation Characterization and Applications of Nanoparticles
}

\author{
Lohat Simranjeet Kaur *, Kumar Sandeep, Gaba Punam \\ Department of Pharmaceutics, Amar Shaheed Baba Ajit Singh Jujhar Singh Memorial College of Pharmacy, Bela (Ropar), India
}

\begin{abstract}
For the past few decades, there has been a major investigate intrigues among the zone of medicate conveyance utilizing particulate conveyance frame works as carriers for the limited and expansive particles. Particulate frameworks like nanoparticles are utilizing as a physical approach to alter and move forward the pharmacological medicine and pharmacodynamics properties of various forms of sedate atoms. Nano particles area unit characterized as a particles with a breadth littler than $100 \mathrm{~nm}$, area unit increasingly utilize in many applications, count medicate framework and to pass organ obstructions like the blood brain barrier. Nano crystals and alternative nanoparticles are obtain ing a parcel of thought for potential utilize in medicine, and medicine sedate revelation. The use of engineering science and prescription drugs and additional significantly sedate connivance is ready to unfold quickly. Right away various substances area unit at a lower place examination for sedate provides.
\end{abstract}

Keywords: Nanoparticles, Types, Method of Preparation, Evaluation, Applications.

Article Info: Received 07 Oct 2020; $\quad$ Review Completed 16 Nov 2020; $\quad$ Accepted 21 Nov 2020; Available online 15 Dec 2020

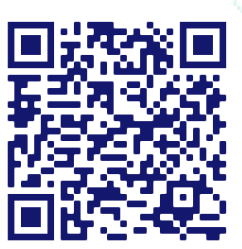

Cite this article as:

Lohat SK, Kumar S, Gaba P, An Overview: Preparation Characterization and Applications of Nanoparticles, Journal of Drug Delivery and Therapeutics. 2020; 10(6-s):159-167 http://dx.doi.org/10.22270/jddt.v10i6-s.4398

*Address for Correspondence:

Pharmacy, Bela (Ropar)

Simranjee t Kaur Lohat Department of Pharmaceutics, Amar Shaheed Baba Ajit Singh Jujhar Singh Memorial College of

\section{INTRODUCTION}

Nanotechnology characterized as a modest science. Nanotechnology the part of innovation that manages measurements and resistances of under 100 nanometers, particularly the control of individual iotas and particles. Nanotechnology includes imaging, estimating, demonstrating, and controlling issue at this length scale. A nanometer is one-billion of a meter. A sheet of paper is around 100,000 nanometers thick; a solitary gold molecule is about $33 \%$ of a nanometer in distance across.

Nanoparticles are particles that exist on a nanometer scale (i.e., beneath $100 \mathrm{~nm}$ in any event one measurement). They can have physical properties, for example, consistency, conductance or unique optical properties that make them attractive in materials science and science. Nanoparticles (NPs) are characterized as particulate scatterings or strong particles medicate transporter that might be biodegradable. The medication is broken down, ensnared, exemplified or connected to a nanoparticle grid. The term nanoparticle is a consolidated name for both nanospheres and nanocapsules. Nano spheres are matrix system in which drug is entrapped inside the cavity as a core material. Nanocapsules are a system where core material is encapsulated inside the cavity but surrounded by unique polymer membranes. ${ }^{\mathbf{1 - 2}}$
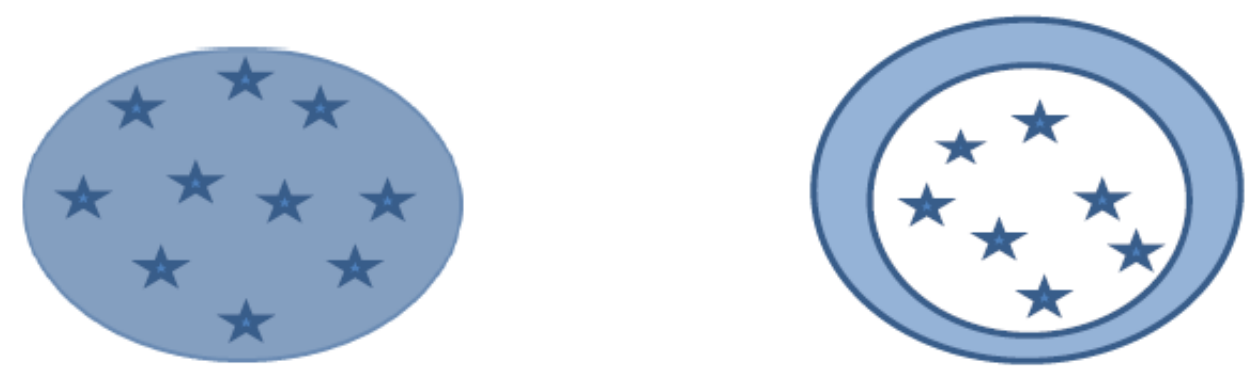

Figure 1 Diagrammatic representation of Types of nanoparticles 


\section{ADVANTAGES OF NANOPARTICLES 3-7}

1. Nanoparticles are control and continue discharge structure at the site oflocalization.

2. Nanoparticles enhance drug circulation in blood, bioavailability, reduce sideeffects.

3. Nanoparticles can be controlled by different courses including oral, nasal, parenteral, intra-visual.

4. In the small regions of body nanoparticles shows better medication as contrast with other dosage form and focus to a specific cell type or receptor.

5. Due to little molecule size nanoparticles beat obstruction by physiological barriers in the body and easily penetrates to cell dividers (cell wall), veins, stomach ,epithelium and blood-cerebrum boundary.
Journal of Drug Delivery \& Therapeutics. 2020; 10(6-s):159-167

6. As a targeted drug carrier nanoparticles reduce drug toxicity and enhance efficient drug distribution.

7. Useful to analyze different diseases.

8. Enhanced stability of ingredients.

9. Prolonged shelf-life.

10. Change the method of drug delivery to improve customer acceptance.

\section{TYPES OF NANOPARTICLES 8}

Nanoparticles can be ordered into various kinds as indicated by the size, morphology, physical and concoction properties.

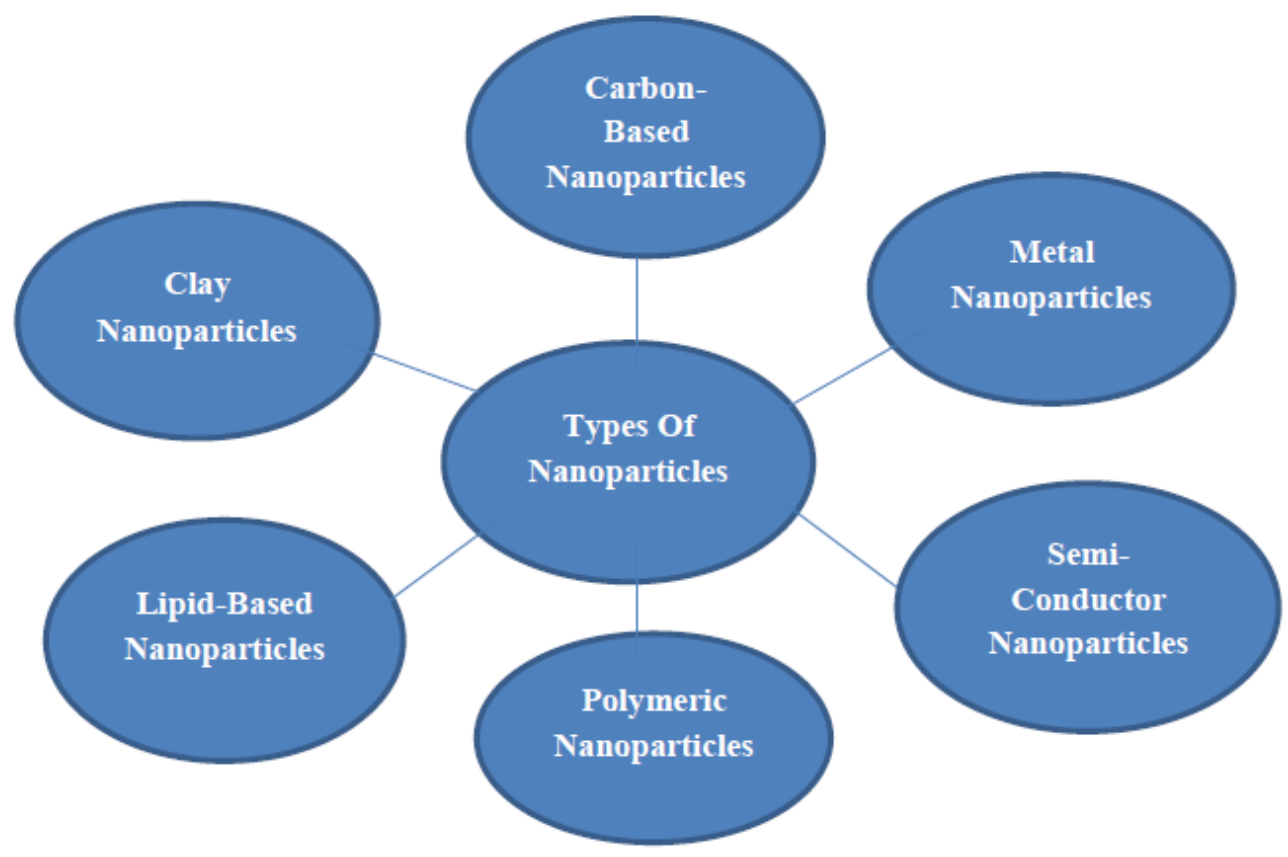

Figure 2: Diagrammatic representation of Types of nanoparticles.

\section{Carbon-based nanoparticles}

Carbon-based nanoparticles incorporate two primary materials: carbon nanotubes (CNTs) and fullerenes. CNTs are only graphene sheets folded into a cylinder. These materials are for the most part utilized for the basic fortification as they are multiple times more grounded than steel.

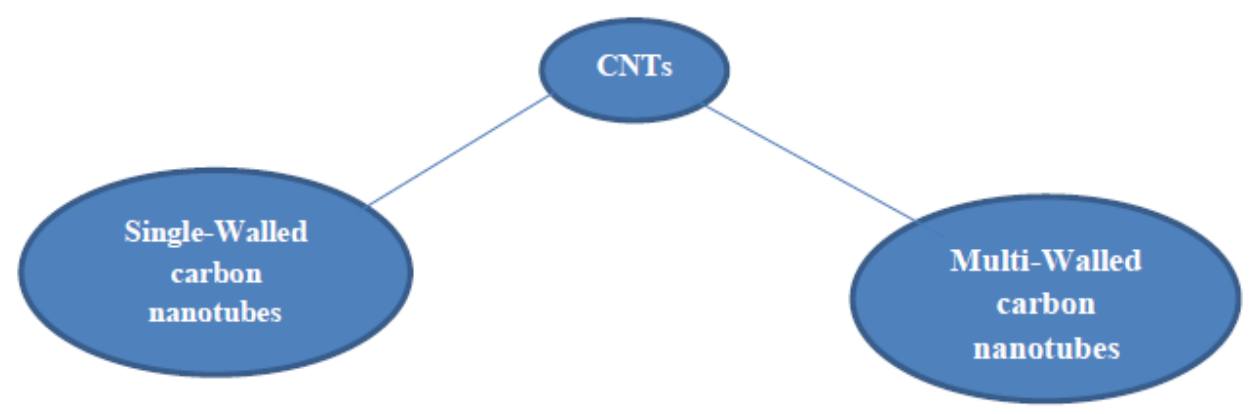

Figure 3: Diagrammatic representation of Types of carbon baesd nanoparticles.

CNTs can be classified into single-walled carbon nanotubes (SWCNTs) and multi-walled carbon nanotubes (MWCNTs). CNTs are remarkable in a manner as they are thermally conductive along the length and non- conductive over the cylinder.
Fullerenes are the allotropes of carbon having a structure of empty confine of at least sixty carbon particles. The structure of C-60 is called Buckminster fullerene, and resembles an empty football. The carbon units in these structures have a pentagonal and hexagonal game plan. 


\section{Lohat et al}

These have business applications because of their electrical conductivity, structure, high quality, and electron partiality.

\section{Clay Nanoparticles/Ceramic Nanoparticles}

Clay nanoparticles are inorganic solids comprised of oxides, carbides, carbonates and phosphates. These nanoparticles have high warmth obstruction and compound latency. They have applications in photocatalysis, photodegradation of colors, sedate conveyance, and imaging.

By controlling a portion of the attributes of fired nanoparticles like size, surface territory, porosity, surface to volume proportion, and so on, they execute as a decent medication conveyance specialist. These nanoparticles have been utilized successfully as a medication conveyance framework for various ailments like bacterial contaminations, glaucoma, malignancy, and so on.

\section{Metal Nanoparticles}

Metal nanoparticles are set up from metal antecedents (metal precursors). These nanoparticles can be blended by concoction, electrochemical, or photochemical techniques. In concoction techniques, the metal nanoparticles are gotten by lessening the metal-particle forerunners in arrangement by substance decreasing specialists. These can adsorb little atoms and have high surface vitality. These nanoparticles have applications in inquire about zones, recognition and imaging of biomolecules and in ecological and bio analytical applications. For instance gold nanoparticles are utilized to cover the example before breaking down in SEM. This is typically done to improve the electronic stream, which encourages us to get excellent SEM pictures.

\section{Semiconductor Nanoparticles}

Semiconductor nanoparticles have properties same as of metals and non-metals. They are found in the occasional table in bunches II-VI, III-V or IV-VI. These particles have wide bandgaps, which on tuning shows various properties. They are utilized in photocatalysis, gadgets, photograph optics and water parting applications. A few instances of semiconductor nanoparticles are GaN, GaP, InP, InAs from bunch III-V, ZnO, ZnS, CdS, CdSe, CdTe are II-VI semiconductors and silicon and germanium are from bunch IV.

\section{Polymeric Nanoparticles}

Polymeric nanoparticles are natural based nanoparticles. Contingent on the technique for readiness, these have
Journal of Drug Delivery \& Therapeutics. 2020; 10(6-s):159-167 structures formed like nanocapsular or nanospheres. A nanosphere molecule has a grid like structure though the nanocapsular molecule has center shell morphology. In the previous, the dynamic mixes and the polymer are consistently scattered while in the last the dynamic mixes are kept and encompassed by a polymer shell.

A portion of the benefits of polymeric nanoparticles are controlled discharge, insurance of medication particles, capacity to consolidate treatment and imaging, explicit focusing on and some more. They have applications in sedate conveyance and diagnostics. The medication conveyances with polymeric nanoparticles are profoundly biodegradable and biocompatible.

\section{Lipid Nanoparticles}

Lipid nanoparticles are commonly circular fit as a fiddle with a distance across extending from 10 to $100 \mathrm{~nm}$. It comprises of a strong center made of lipid and a lattice containing dissolvable lipophilic atoms. The outer center of these nanoparticles is balanced out by surfactants and emulsifiers. These nanoparticles have application in the biomedical field as a medication bearer and conveyance and RNA discharge in malignant growth treatment.

Hence, the field of nanotechnology is a long way from being immersed and it is, as the measurement says, sitting on the staircase of an exponential development design. It is fundamentally at a similar stage as the data innovation was during the 1960 and biotechnology in the time of 1980 . Along these lines it can undoubtedly be anticipated that this field would observer an equivalent exponential development as the other two innovative field saw before.

\section{METHODS OF PREPARATION}

\section{Solvent Evaporation Method 9-12}

In this strategy, polymer is broken down in natural dissolvable (dichloromethane, chloroform or ethyl acetic acid derivation) and afterward the drug is scattered in this arrangement. At that point this blend is emulsified in a aqueous stage containing surfactant (polysorbates, polaxomers, poly vinyl liquor) to make an oil in water $(0 / W)$ emulsion by utilizing mechanical mixing, sonication or miniaturized scale fluidization. After the arrangement of an emulsion the natural dissolvable is dissipated by expanding the temperature and utilizing Fig. 4 Diagrammatic representation of Solvent Evaporation Method.

\section{Organic solvent+ Polymer +Drug}

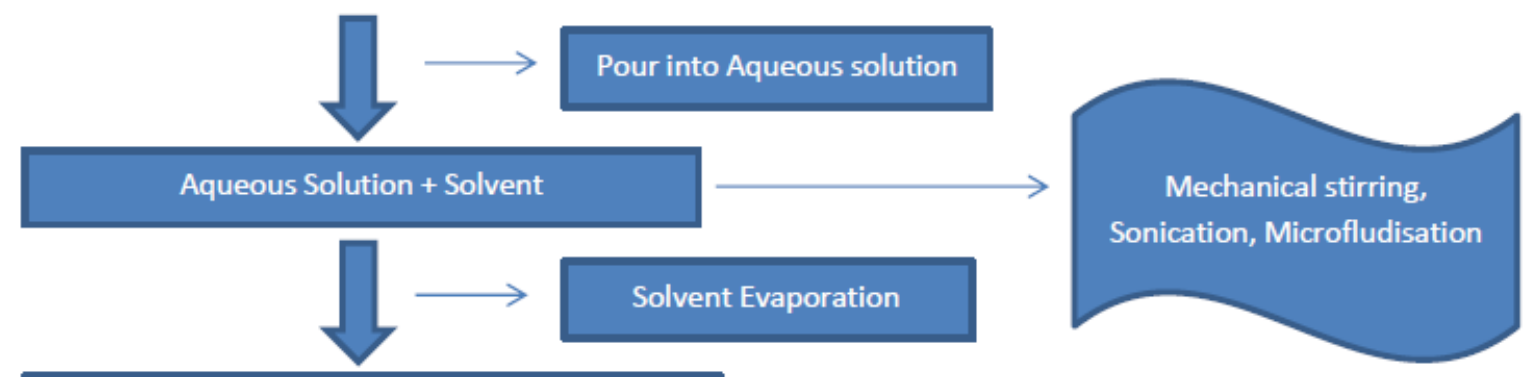

Nanoparticles

Figure 4: Diagrammatic representation of Solvent Evaporation Method 


\section{Solvent Diffusion method 13-16}

In this technique, the medication and lipid are broken up in natural, water miscible solvents at raised temperature and the resultant arrangement are quickly infused into a fluid stage containing surfactant, under mechanical mixing. As the temperature brings down (cools), the lipid beads cement and the nanoparticles suspension of the medication are framed. This strategy has pulled in incredible enthusiasm because of its effortlessness and simplicity of taking care of.

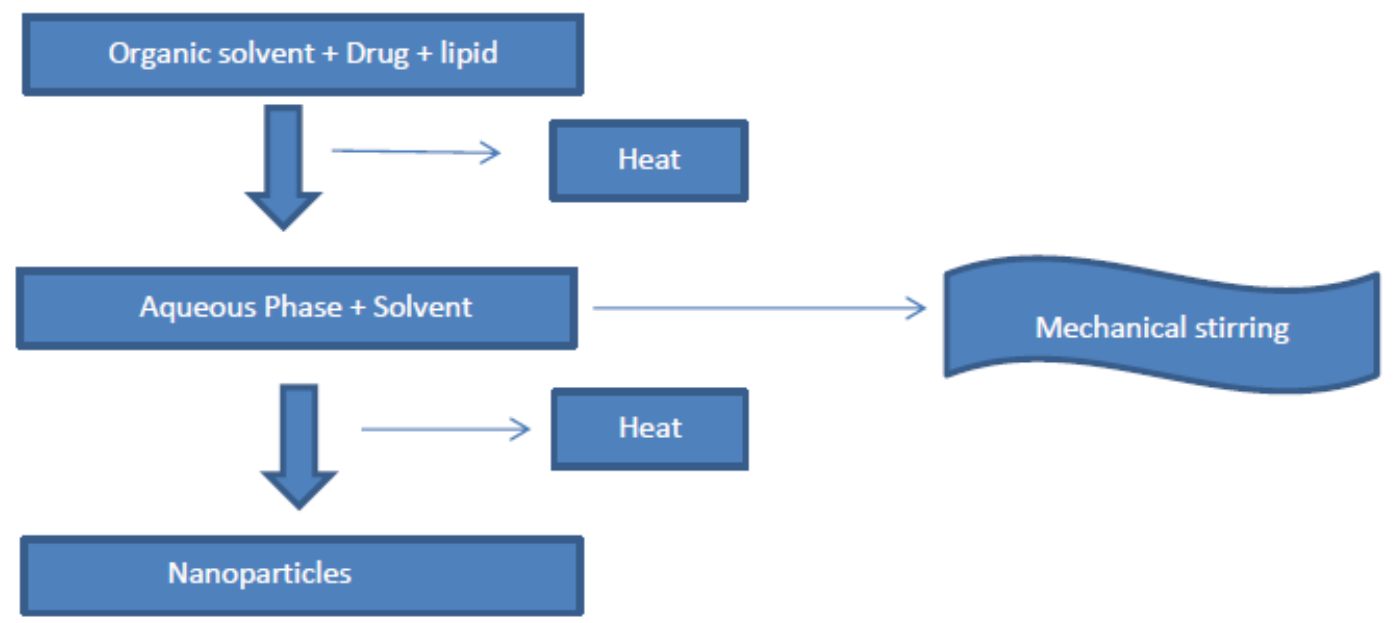

Figure 5: Diagrammatic representation of Solvent Diffusion Method

\section{Nano precipitation method without surfactant 17-19}

This technique is most appropriate for water insoluble medications. Medication arrangement (lyophobic) is blended in with against dissolvable under attractive mixing. Accelerates are shaped rapidly and after that nanoparticles were separated and afterward item is lyophilized.
The size and medication discharge is constrained by altering readiness parameters. Elements which impact size of nanoparticles are:-expansion of natural stage into fluid stage and Altering grouping of polymer. Thus method is shown in fig.6.

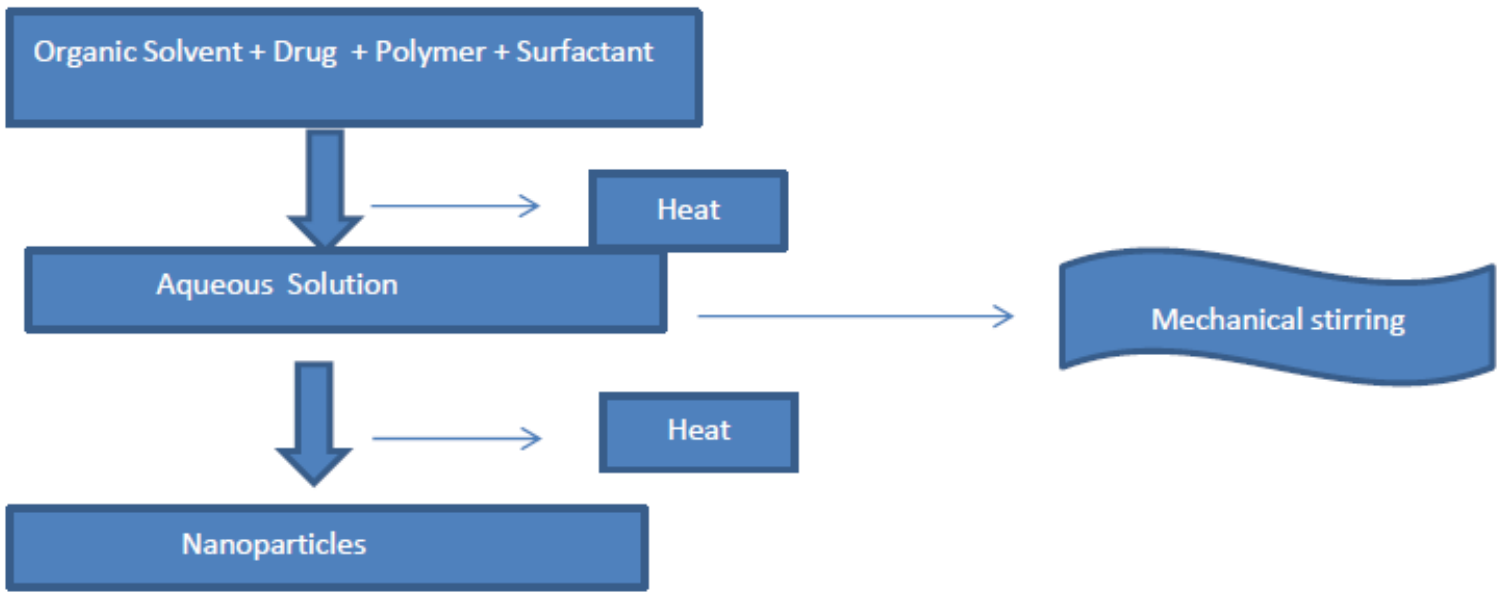

Figure 6: Diagrammatic representation of Nano-precipitation method without surfactant

\section{Ionic Gelation Method or Coacervation Method 20-23}

In this strategy, hydrophilic polymers are utilized which are having biodegradable nature, for example, Chitosan, gelatin and Sodium alginate. In this strategy we need a blend of two fluid stages, and the first is chitosan polymer and the other one is polyanion tripolyphosphate. Chitosan is having a decidedly charged amino gathering and tripolyphosphate
(TPP) having a negative charge bunch so due to inverse charges on the two polymers they interface with one another and structure coacervates in the scope of nanometers. In Ionic gelation, material undergoing transition from liquid to gel at room temperature. This Method shown in fig.7 
Chitosan + Drug Solution

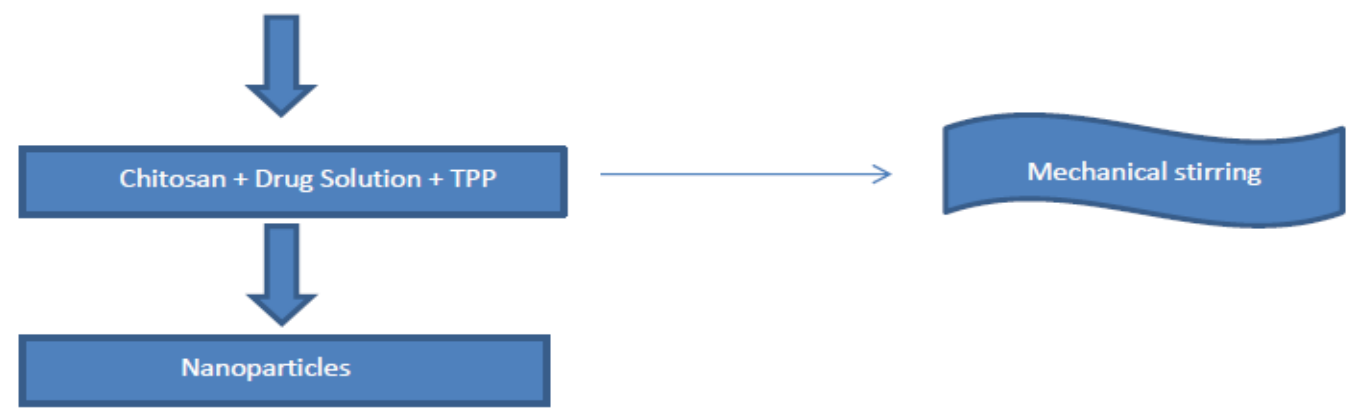

Figure 7: Diagrammatic representation of Ionic Gelation Method or Coacervation Method

\section{Supercritical Fluid Technology 24}

Supercritical liquids are earth safe so they have no unsafe impact which happens in the event of different techniques in light of the fact that in different strategies natural solvents are included. Supercritical fluid is defined as a solvent at a temperature above its critical temperature, at which the fluid remains a single phase regardless of pressure. They are broadly utilized due to non-danger, non-combustibility and low cost. In this technique $\mathbf{C O 2}$ is generally favored as a supercritical liquid as a result of its mellow conditions. This supercritical liquid is utilized in two primary systems and the first is Supercritical enemy of Solvent and other one is Rapid development of basic arrangement (RESS).

1. In Supercritical Anti-Solvent procedure solvents are utilized which are fluids in nature and which ought to totally solubilize with supercritical liquid. Ex. methanol is utilized as dissolvable which is miscible with the supercritical liquid, the concentrate of the dissolvable by supercritical liquid prompts the momentary precipitation of the solute which results development of particles in nano.

2. Rapid extension of basic arrangement (RESS) the method is utilized to create bio-erodible polymeric microspheres stacked with a helpful medication, and this definition is appropriate for controlled conveyance applications. In RESS, Solute (Drug) is broken down in a supercritical liquid to shape an answer. At that point this arrangement is quickly extended over an opening or a fine spout into surrounding air by the fast weight decrease in the development. These outcomes in homogenous nucleation and in this way development of very much scattered nanoparticles. It is best procedure since it is dissolvable free.

\section{High Pressure Homogenisation 25-27}

In this technique the medication is premilled by utilizing low weight homogenizer and afterward includes fluid arrangement of surfactant at various degrees of fixations. Medication is broken down in lipid stage (liquefied) and this arrangement was then disintegrated in ethanol. The natural arrangement is then moved to the watery surfactant arrangement under blending at $500 \mathrm{rpm}$ for $20 \mathrm{~min}$ utilizing a mechanical stirrer. These outcomes into development of a pre-emulsion which is then therefore homogenized to diminish the size utilizing high weight homogenizer. At long last, the blend is cooled to room temperature yielding medication nanoparticles.
Lipid phase + Drug (Organic Solution)

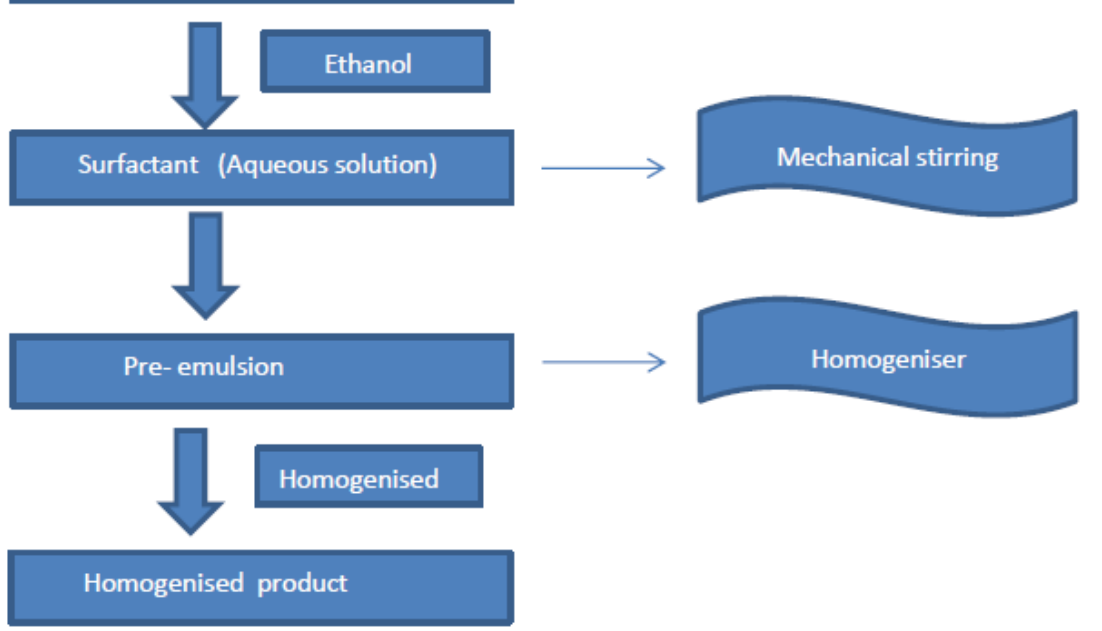

\section{Cool at RT}




\section{CHARACTERIZATION OF NANOPARTICLES}

Nanoparticles are generally characterized by their size, morphology and surface charge, using such advanced microscopic techniques as scanning electron microscopy (SEM), transmission electron microscopy (TEM) and atomic force microscopy (AFM).

\section{Yield of Nanoparticles 28}

The yield of nanoparticles was dictated by looking at the entire load of nanoparticles framed against the combined load of the copolymer and drug.

$$
\% \text { yield }=\quad \frac{\text { Amount of Nanoparticle }}{\text { Amount of Drug }+ \text { Polymer }} \times 100
$$

\section{Drug Content / Surface entrapment / Drug entrapment 29}

After centrifugation measure of medication present in supernatant $(\mathrm{w})$ controlled by UV spectrophotometery. After that standard adjustment bend plotted. At that point measure of medication present in supernatant subtracted from the aggregate sum utilized in the planning of nanoparticles $(\mathrm{W}) .(\mathrm{W}-\mathrm{w})$ is the measure of medication entangled. \% tranquilize entanglement determined by

$$
\% \text { Drug Entrapment }=\frac{w-w}{w} \times 100
$$

\section{Polydispersity index ${ }^{30}$}

Polydispersity index of prepared nanoparticles was carried out by using Malvern Zetasizer.

\section{Kinetic Study 31}

For estimation of the motor and system of medication discharge, the aftereffect of in vitro medication discharge investigation of nanoparticles were fitted with different active condition like zero request (combined \% discharge versus time), first request (log \% tranquilize remaining versus time), Higuchi's model (aggregate \% sedate discharge versus square base of time). $\mathrm{R}^{2}$ and $\mathrm{k}$ esteems were determined for the direct bend got by relapse investigation of the above plots.

\section{Stability of Nanoparticles 32}

Stability studies of prepared nanoparticles determined by storing optimized formulation at $4^{\circ} \mathrm{C} \pm 1^{\circ} \mathrm{C}$ and $30^{\circ} \mathrm{C} \pm 2^{\circ} \mathrm{C}$ in stability chamber for 90 days. The samples were analyzed after a time period like at $0,1,2$, and 3 months for their drug content, drug release rate ( $\mathrm{t} 50 \%)$ as well as any changes in their physical appearance.

\section{Particle size 33}

Molecule size distribution and morphology are the most significant parameters of characterization of nanoparticles. Morphology and size are estimated by electron microscopy. The significant utilization of nanoparticles is in drug discharge and medication focusing on. It has been discovered that molecule size influences the drug discharge. Smaller particles offer larger surface area. Thus, a large portion of the medication stacked onto them will be exposed to the molecule surface leading to quick medication discharge. Despite what might be expected, drug gradually diffuses inside bigger particles. As a disadvantage, smaller particles tend to aggregate during storage and transportation of nanoparticle dispersion. Thus, there is a tradeoff between a little size and most extreme strength of nanoparticles.

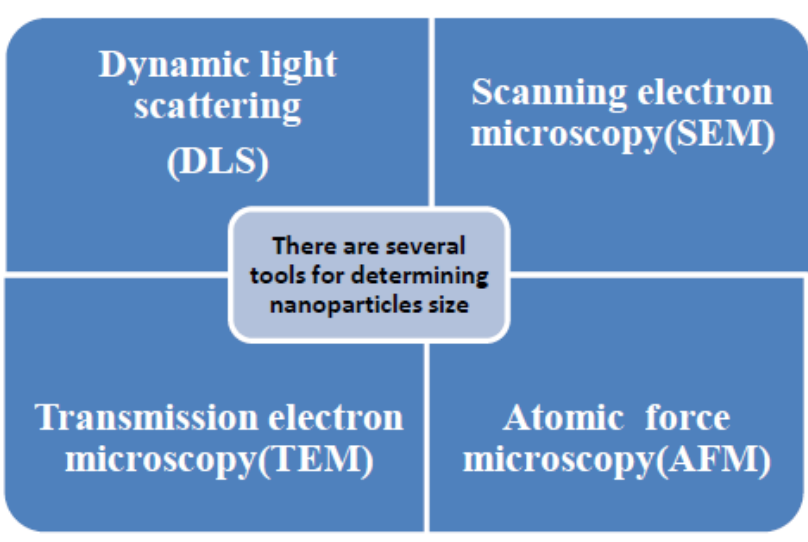

Figure 9: Tools for determining nanoparticles size

\section{Dynamic light scattering (DLS): 34}

Currently, the fastest and most popular method of determining particle size is dynamic light scattering (DLS). It is also known as the photon-correlation spectroscopy (PCS). DLS is generally used to decide the size of Brownian nanoparticles in colloidal suspensions in the nano and submicron ranges. Sparkling monochromatic light (laser) onto an answer of round particles in Brownian movement causes a Doppler move when the light hits the moving molecule, changing the wavelength of the approaching light. This change is identified with the size of the molecule. It is conceivable to extricate the size circulation and give a portrayal of the molecule's movement in the medium, estimating the dissemination coefficient of the molecule and utilizing the autocorrelation work. The photon connection spectroscopies (PCS) speak to the most much of the time utilized method for precise estimation of the molecule size and size circulation dependent on DLS.

\section{Scanning Electron microscopy: $35-38$}

Filtering electron microscopy (SEM) is giving morphological assessment with direct perception. The strategies dependent on electron microscopy offer a few points of interest in morphological and estimating investigation; be that as it may, they give restricted data about the size dispersion and genuine populace normal. For SEM portrayal, nanoparticles arrangement ought to be first changed over into a dry powder, which is then mounted on an example holder pursued by covering with a conductive metal, for example, gold, utilizing a sputter coater. The example is then examined with a concentrated fine light emission .The surface attributes of the example are acquired from the auxiliary electrons transmitted from the example surface. The nanoparticles must have the option to withstand vacuum, and the electron pillar can harm the polymer. The mean size acquired by SEM is tantamount with results gotten by powerful light dispersing. Additionally, these systems are tedious, expensive and oftentimes need corresponding data about measuring distribution.

\section{Transmission electron microscope: 39}

TEM works on startling rule in comparison to SEM, however it habitually brings same sort of data. The test planning for TEM is complex and time expending since of its necessity to be ultra lean for the electron transmittance. The nanoparticles scrambling is kept onto reinforce systems (back networks) or movies. To form nanoparticles withstand the instrument vacuum and empower taking care of, they are settled utilizing either a negative recoloring fabric, for illustration, phosphotungstic destructive or backups, uranyl acidic corrosive induction, and so forward, or by plastic introducing. Substitute technique is to open the example to 
liquid nitrogen temperatures within the wake of embedding in vitreous ice. The surface characteristics of the test are gotten when a bar of electrons is transmitted through an ultra lean test, connection with the test because it passes through.

\section{Atomic force microscopy: $\mathbf{4 0 - 4 2}$}

Atomic force microscopy (AFM) offers ultra-high goals in molecule size estimation and depends on a physical examining of tests at sub-micron level utilizing a test tip of nuclear scale. Instrument gives a geological guide of test dependent on powers between the tip and the example surface. Tests are generally filtered in contact or noncontact mode relying upon their properties. In contact mode, the geographical guide is created by tapping the test on to the surface over the example and test drifts over the directing surface in non-contact mode. The prime bit of leeway of AFM is its capacity to picture non-leading examples with no particular treatment, thus permitting imaging of sensitive organic and polymeric nano and microstructures. AFM gives the most exact portrayal of size and size circulation and requires no scientific treatment. In addition, molecule size obtained by AFM strategy gives genuine picture which comprehends the impact of different organic conditions.

\section{Surface Charge: 43}

The nature and intensity of the surface charge of nanoparticles is significant as it decides their connection with the organic condition just as their electrostatic cooperation with bioactive mixes. The colloidal dependability is analyzed through zeta capability of nanoparticles. This potential is a circuitous proportion of the surface charge. It relates to potential distinction between the external Helmholtz plane and the outside of shear. The estimation of the zeta potential takes into consideration expectations about the capacity soundness of colloidal scattering. High zeta potential qualities, either positive or negative, ought to be accomplished so as to guarantee security and maintain a strategic distance from total of the particles. The degree of surface hydrophobicity would then be able to be anticipated from the estimations of zeta potential. The zeta potential can likewise give data in regards to the idea of material typified inside the nano capsules or covered onto the.

\section{Surface hydrophobicity: 44}

Surface hydrophobicity can be controlled by a few systems, for example, hydrophobic connection chromatography, biphasic parceling, adsorption of tests, contact angle estimations and so forth. As of late, a few modern diagnostic systems are accounted for in writing for surface examination of nanoparticles. X-beam photon relationship spectroscopy allows the identification of specific chemical groups on the surface of nanoparticles.

\section{Drug Release: $45-48$}

A focal purpose behind seeking after nanotechnology is to convey drugs, henceforth understanding the way and degree to which the medication particles are discharged is significant. So as to get such data most discharge strategies necessitate that the medication and its conveyance vehicle be isolated. The medication stacking of the nanoparticles is commonly characterized as the measure of medication bound per mass of polymer (typically moles of medication per mg polymer or mg tranquilize per mg polymer); it could likewise be given as rate comparative with the polymer. The procedure utilized for this examination is traditional explanatory strategies like UV spectroscopy or elite fluid chromatography (HPLC) after ultracentrifugation, ultra filtration, gel filtration, or radiating ultrafiltration. Measurement is performed with the UV spectroscopy or HPLC. Medication discharge tests are additionally like medication stacking examines which is evaluated for a while to break down the instrument of medication discharge.

In-vitro medicate discharge thinks about were performed in USP Type II disintegration mechanical assembly at pivot speed of $50 \mathrm{rpm}$. The readied inundated in $900 \mathrm{ml}$ of phosphate cradle arrangement in a vessel, and temperature was kept up at $37 \pm 20^{\circ} \mathrm{C}$. Required amount $5 \mathrm{ml}$ of the medium was pulled back at explicit timespans and a similar volume of disintegration medium was supplanted in the cup to keep up a steady volume. The pulled back examples were dissected utilizing UV spectrophotometer.

\section{APPLICATIONS OF NANOPARTICLES 49}

Applications of nanotechnology in the different field are represented below;

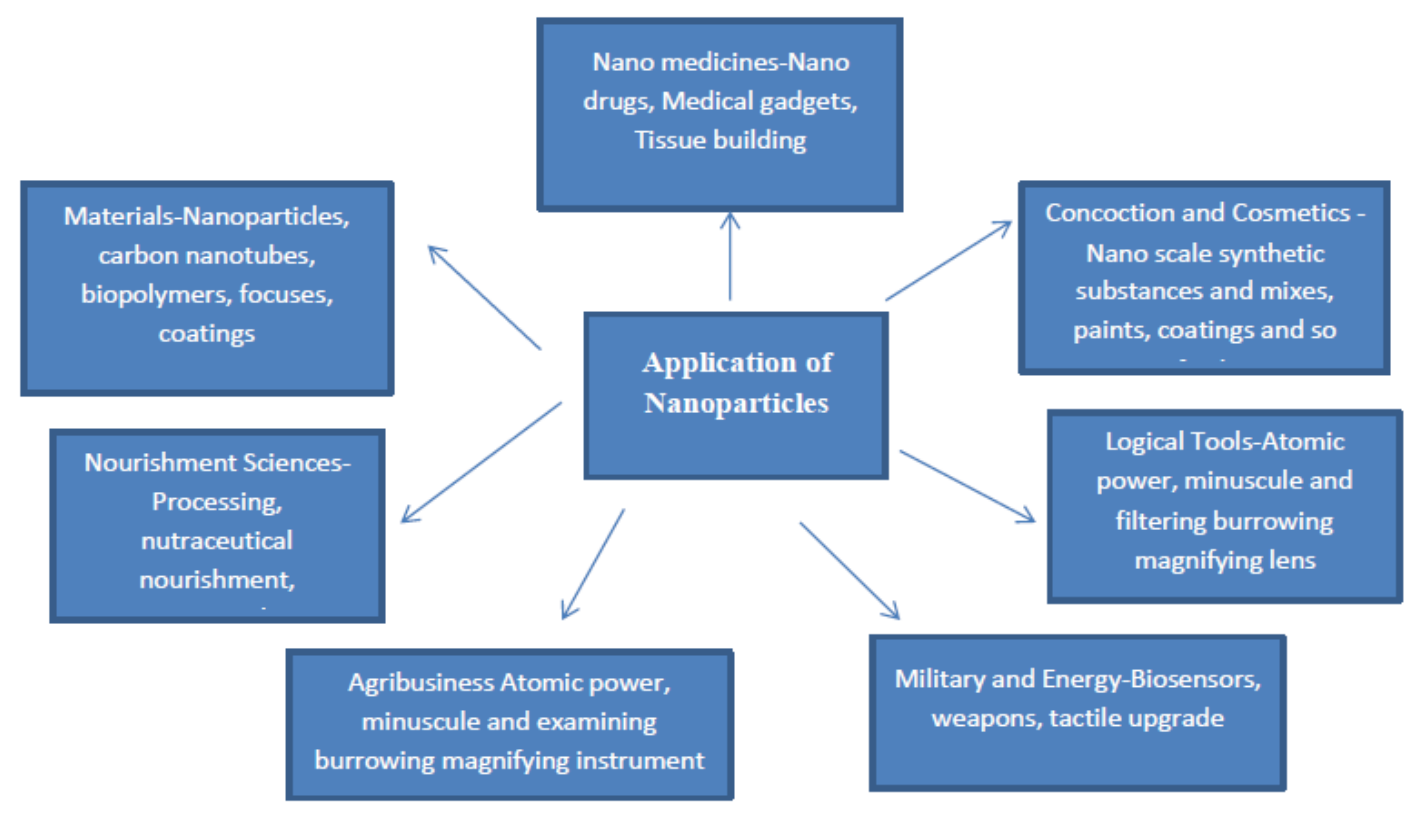

Figure 10: Tools for determining nanoparticles size 


\section{HEALTH IMPLICATION OF NANOPARTICLES 50}

It is essential to separate among 'free' and 'fixed' nano particles. The formers represent an immediate wellbeing risk since they are progressively hard to contain because of airborne and can be breathed in. Nanoparticles can enter the human body in a few different ways (i) by means of the lungs where a quick translocation through the circulatory system to essential organ is conceivable, including crossing the blood brain barrier and ingestions by

\section{(ii) the intestinal tract (iii) the skin}

Lungs: Based on three molecule types titanium dioxide (Ti02) carbon dark and the diesel particles, dangers considers in rodents, exhibit that ultrafine nanoparticles organization to the lung produce progressively intense unfriendly impact as irritation and resulting tumors contrasted and bigger measured particles, of indistinguishable concoction piece at proportionate mass fixation. Surface properties, for example, surface science may assume a critical job in nanoparticles poisonous quality. 51-53

Intestinal Tract: The epithelium of the little and huge intestinal is in close contact with ingested material so supplements can be used. A blend of disaccharides, peptides, unsaturated fats and monoglycerides produced however assimilation in small digestive system are additionally changed and taken in the villi. Charged particles like carboxylated polystyrene nano particles or those made out of emphatically charged polymer show poor oral bioavailability through electrostatic aversions and means ensnarement. The littler the particles width the quicker they could enter the bodily fluid to arrive at the colonic eutrocytes; $14 \mathrm{~nm}$ breadth penetrated inside 2 mints, $415 \mathrm{~nm}$ particles look 30 mints while $1000 \mathrm{~nm}$ particles were not able translocate this barrier. 54-55 $^{-5}$

Skin: Particles 500-1000 nm in size hypothetically past the domains of nano innovation can infiltrate and arrive at the lower levels of human skin, 128 and littler particles are probably going to increasingly more profound into the skin 56.

\section{CONCLUSION:}

Nanotechnology is opening imminent future in pharmaceutics. Nanoparticle is novel methodology for medicate conveyance which we can accomplish better restorative activity, better bioavailability, decrease poisonous quality. Today nanoparticles are effectively utilized in cerebrum focusing, in disease treatment and soon nanoparticles offers us a chance to improve understanding consistence for better therapy.

\section{ACKNOWLEDGEMENT}

Amar Shaheed Baba Ajit Singh Jujhar Singh Memorial Collage of Pharmacy, Bela (Ropar)

\section{CONFLICT OF INTERESTS}

The author(s) confirm that this article content has no conflict of interest

\section{REFERENCES:}

1. Lal Pal S, Jana U, Manna P. K., Mohanta G. P., Manavalan R:Nanoparticle: An overview of preparation and characterization. Journal of Applied Pharmaceutical Science 2011; 1:6: 228-234.

2. Khosla G, Goswami L, Kothiyal P, Mukhopadhyay S. Nanoparticles: A Novelistic Approach for CNS disorders. Journal of Advanced Pharmaceutical Sciences 2012; 2:2: 220-259.
3. Abhilash M: Potential applications of Nanoparticles International Journal of Pharma and Bio Sciences 2010; 1:1: 112.

4. Mullaicharam A.R: Nanoparticles in drug delivery system. International Journal of Nutrition, Pharmacology Neurological Diseases 2011; 1:2: 103-121.

5. Vyas SP, Khar RK. Targeted and controlled drug delivery. CBS publishers and distributers. New Delhi ocfx 2002; 1:331-43.

6. Pangi Z, Beletsi A. Even gelatos K. PEG-ylated nanoparticles for biological and pharmaceutical application. Adv drug Del rev. 2003; 24: 403-19.

7. Anilkumar J Shinde, and Harinath N. Nanoparticles - As Carriers for Drug Delivery System. 2009; 3(4): 80-86.

8. https://www.azonano.com/article.aspx?ArticleID=4938\#: : $\operatorname{targ}$ etText=Nanoparticles $\% 20$ can $\% 20 \mathrm{be} \% 20$

classified $\% 20$ into,nanoparticles $\% 20$ and $\% 20$ lipid $\% 2$ Dbased $\% 2$ 0nanoparticles.

9. Nikam Arti P., Mukesh Ratnaparkhiand P., Chaudhary Shilpa P: Nanoparticles-an overview, Int. J. Res.Dev. Pharm. L. Sci. 2014; 3(5): 1121-1127.

10. Kaparissides C, Alexandridou S, Kotti K, Chaitidou S: Recent advances in novel drug delivery systems 2006; 2026-2027.

11. Kohler Fritzsche W: Nanotechnology. An introduction to Nanostructuring Koosha F, Muller RH, Davis SS, Davies MC. The surface chemical structure of poly (-hydroxybutyrate) microparticles produced by solvent evaporation process. J Control Release 1989; 9:149-57.

12. Desgouilles St, Vauthier C, Bazile D, Joe"l Vacus, Grossiord JeanLouis, Veillard Mi, and Patrick Couvreur: The Design of Nanoparticles Obtained by Solvent Evaporation. A Comprehensive Study Langmuir 2003; 19: 9504-9510.

13. Ueda $M$, Kreuter J. Optimization of the preparation of loperamide- loaded poly (l-lactide) nanoparticles by high pressure emulsification solvent evaporation. J Microencapsul. 1997; 14: 593-605.

14. Nagavarma, B.V.N, Hemant, K.S, Yadav, Ayaz, Vasudh, L.S, Shivakumar, H.G. Different techniques for preparation of polymeric nanoparticles- a review. Asian J. Pharm. Clin. Res. 2012; 5(3): 16-23.

15. Hu FQ ,Yuan H, Jiang SP, Du YZ, Ye YQ, Zeng S: Preparation and Characterisation of stearic acid nanostructured lipid carriers by solvent diffusion method in an aqueous system. Colloids and Surfaces B Biointerfaces 2005; 45(3): 167-173.

16. Lal pal S, Jana U, Manna P.K., Mohanta G.P., Manavalan R: Nanoparticles: An overview of preparation and characterization. Journal of Applied Pharmaceutical Science 2001; 01 (06):228234.

17. Quintanar-Guerrero D, Allemann E, Fessi H, Doelker E: Preparation techniques and mechanism of formation of biodegradable nanoparticles from preformed polymers. Drug Dev Ind Pharm 1998;24: 113-128.

18. Prathna T.C., Lazar Mathew N., Chandrasekaran, Ashok M. Raichur, Amitava Mukherjee: Biomimetic synthesis of nanoparticles: science, technology \& applicability. In: Amitava Mukherjee (Ed.), Biomimetics, learning from nature. Published by InTechOpen 2010.

19. Iravani S, Zolfaghari B: Green synthesis of silver nanoparticles using Pinus eldarica bark extract. Hindawi Publishing Corporation, Biomed. Res. Int. 2013; 1-5.

20. Bilati U, Eric All emann, Eric Doelker. Development of a nanoprecipitation method intended for the entrapment of hydrophilic drugs into nanoparticles. European Journal of Pharmaceutical Science 2005; 24(1):67-75.

21. Prasad Rao, J., Kurt Geckeler, E. Polymer nanoparticles: Preparation techniques and size-control parameters. Prog. Polymer Sc. 2011; 36(7): 887-913.

22. Gelation preparation technique Umasankar K, Uma Maheswara Reddy C. Formulation and Evaluation of Cytarabine Nanoparticles. International Journal of Innovative Pharmaceutical Research 2010; 1(2):48-52.

23. Katherine Bowman, Kam W Leong. Chitosan Nanoparticles for Oral drug and Gene Delivery. Int J of Nanomedicine 2006; 1(2): 117-128.

24. Sailaja et al. Different Techniques for the preparation of nanoparticles using natural polymers and their applications. Int J Pharm Sci. 2011; 3 (2): 45-50.

25. Renu T. A Review on nanoparticles-preparation and evaluation parameters. Indian Journal of Pharmaceutical and Biological Research (IJPBR) 2015; 4(2): 27-31. 
26. Tripura SP. Et al. Formulation and Evaluation of Fabuxostat Nanoparticles. American Journal of Pharm Tech research 2014; 4(1): 2249-2287.

27. Yadav N, khatak S, Vir Singh U: Solid lipid nanoparticles- a review. International journal of applied pharmaceutics 2013; 5(2): 8-18.

28. Allemann E, Gurny R, Doekler E: Drug-loaded nanoparticle preparation methods and drug targeting issues. Eur J Pharm Biopharm. 1993; 39:173-91.

29. Lakshmana Prabu S, Shirwaikar AA, Shirwaikar A, Kumar A: Formulation and evaluation of sustained release microspheres of rosin containing Aceclofenac, Ars Pharm 2009; 50:2: 5162.

30. Das S, Banerjee $\mathrm{R}$ and Bellare J: Aspirin Loaded Albumin Nanoparticles by Coacervation: Implications in Drug Delivery, Trends Biomater. Artif Organs 2005; 18:2: 1-10.

31. Aejaz A, Azmail K, Sanaullah S and Mohsin A: Formulation and in vitro evaluation of Aceclofenac solid dispersion incorporated gels. International Journal of Applied Sciences 2010; 2:1: 7-12.

32. Tamizhrasi S, Shukla A, Shivkumar T, Rathi V, Rathi J. C: Formulation and evaluation of Lamivudine loaded polymethacrylic acid nanoparticles. International Journal of PharmTech Research [IJPRIF] 2009; 1:3: 411-415.

33. Mukhopadhyay S, Madhav Satheesh N.V. and Upadhyaya K: Formulation and evaluation of bionanoparticulated drug delivery of Rivastigmine. World Journal of Pharmaceutical Sciences 2016; 4:5: 264-272.

34. Redhead H M., Davis SS. and Illum L. J Drug delivery in poly(lactide-co-glycolide) nanoparticles surface modified with poloxamer 407 and poloxamine 908: in vitro characterisation and in vivo evaluation Control. Release. 2001; 70: 353.

35. DeAssis DN., Mosqueira VC., Vilela JM., Andrade M.S., Cardoso VN: Release profiles and morphological characterization by atomic force microscopy and photon correlation spectroscopy of $99 \mathrm{~m}$ Technetium - fluconazole nanocapsules. Int J Pharm. 2008; 349: 152 - 160 .

36. Jores K., Mehnert W., Drecusler M., Bunyes H., Johan C., MAder K Investigation on the stricter of solid lipid nanopartuicles and oil-loaded solid nanoparticles by photon correlation spectroscopy, field- flow fractionasition and transmission electron microscopy. J Control Release. 2004; 17: 217- 227.

37. Molpeceres J., Aberturas MR., Guzman M: Biodegradable nanoparticles as a delivery system for cyclosporine: preparation and characterization. J Microencapsul. 2000; 17: 599-614.

38. Dolenc A, Govedarica B, Dreu R, Kocbek P, Srčič S, Kristl J: Nanosized particles of orlistat with enhanced in vitro dissolution rate and lipase inhibition. International Journal of Pharmaceutics. 2010; 396(1):149-55. 20

39. Choi, H.K., Jung, J.H., Ryu J.M., Yoon, S.J., Oh, Y.K. and Kim: Development of insitu gelling and mucoadhesive acetamoniphen liquid suppository, Int. J pharm. 1998;165 :3344.

40. Molpeceres J., Aberturas MR., Guzman M: Biodegradable nanoparticles as a delivery system for cyclosporine: preparation and characterization. J Microencapsul. 2000; 17: 599-614.

41. Muhlen AZ., Muhlen EZ., Niehus H., Mehnert W: Atomic force microscopy studies of solid lipid nanoparticles. Pharm Res.
13:14111416(1996).

42. Shi HG., Farber L., Michaels JN., Dickey A., Thompson KC. Shelukar SD., Hurter PN., Reynolds SD., Kaufman MJ: Characterization of crystalline drug nanoparticles using atomic force microscopy and complementary techniques. Pharm Res. 20: 479 - 484 (2003).

43. Polakovic M., Gorner T., Gref R., Dellacherie E: Lidocaine loaded biodegradable nanospheres. II. Modelling of drug release. J Control Release. 1999; 60: 169 -177.

44. Pangi Z., Beletsi A., Evangelatos K. :PEG-ylated nanoparticles for biological and pharmaceutical application. Adv Drug Del Rev. 2003; 24: 403- 419.

45. Scholes PD., Coombes AG., Illum L., Davis SS., Wats JF., Ustariz C., Vert M., Davies MC: Detection and determination of surface levels of poloxamer and PVA surfactant on biodegradable nanospheres using SSIMS and XPS. J control Release 1999; 59: 261 - 278.

46. Anil kumar J. Shinde and Harinath N: Formulation, development and characterization of Simvastatin nanoparticles by solvent displacement method, Der Pharmacia Lettre, 2014; 6:2: 145155.

47. Jaiswal J., Gupta SK., Kreuter J: Preparation of biodegradable cyclosporine nanoparticles by high- pressure emulsification solvent evaporation process. J Control Release. 2004; 96:16921708.

48. Kreuter J: Physicochemical characterization of polyacrylic nanoparticles. Int. J. Pharm. 1983; 14: 43 - 58.

49. Magenhein B., Levy MY., Benita S: A new in vitro technique for the evaluation of drug release profile from colloidal carriers ultrafiltration technique at low pressure. Int. J. Pharm. 1993; 94: 115-123.

50. Hoet PMH., Brnske HI., Salata OR: Nano particles known and unknown health risk. Jnanobiotecnol. 2004; 2:12.

51. Lee KP., Kelly DP., Oneal FO., Kennedy GL: Lung response to ultrafine kertar aramid synthetic fibrils following 2-year inhalation exposure in rats. Findam Appl Toxicol. 1998;11:1-20

52. Mecke A., Uppuluri S., Sassanella TM., Lee DK., Ramamoorthy A. Baker Jr JR: Direct observation of lipid bilayer disruption by poly (amidoamine) dendrimers. Chem Phys Lipids. 2004; 132:314.

53. Yoo HS., Oh JE., Lee KH., Park TG: Biodegradable nanosparticles containing PLGA conjugates for sustained release. Pharm Res. 1999; 16: 1114-1118.

54. Jani P., Halbert GW., Langridge J., Florence AT: Nanoparticles uptake by the rat gastrointestinal mucosa: quantitation and particle size dependency. J Pharm Pharmacol.1990; 42(12):812826.

55. Jani P., Halbert GW., Langridge J., Florence AT: The uptake and translocation of latex nanosphere and microsphere after oral administration to rats. J Pharm Pharmacol.1989; 41(12) 809812.

56. Lademann J., Weigmann H., Rickmeyer C., Barthelmes H., Schaelmes H., Mueller G, Sterry W: Penetration of Titanium Dioxide microparticles in a sunscreen formulation into the horny layer and the follicular orifice. Skin Pharmacology and Applied Skin Physiology1999; 12: 247- 256. 